\title{
Psychological status of lung cancer patients and the effects of online intervention during the coronavirus disease 2019 pandemic
}

\author{
WENQI SUN and JINYOU LI \\ Department of Thoracic Surgery, Affiliated Hospital of Jiangnan University, Wuxi, Jiangsu 214000, P.R. China
}

Received April 28,2020; Accepted April 27, 2021

DOI: $10.3892 /$ wasj.2021.105

\begin{abstract}
The aim of the present study was to evaluate the anxiety and depression status of lung cancer patients during the coronavirus disease 2019 (COVID-19) pandemic and the effects of psychological counseling online. For this purpose, following enrollment. patients were divided into an intervention group (group I) and a control group (group C). Participants in the two groups were scored using the Self-Rating Anxiety Scale (SAS) and Self-Rating Depression Scale (SDS) before and one week after the intervention. The depression and anxiety status of the lung cancer patients during the COVID-19 pandemic was evaluated based on SAS and SDS, and the effects of intervention online were also evaluated. The results revealed that the rate of anxiety in patients with lung cancer reached $35.71 \%$, while the rate of depression reached $51.43 \%$. After one week of intervention, the standard total score of SAS (S-SAS) and the raw sum score of the SDS (R-SDS) in group I were lower than those in group $\mathrm{C}(\mathrm{P}<0.05)$. The S-SAS and $\mathrm{R}-\mathrm{SDS}$ in group I after one week of intervention were lower than those before intervention $(\mathrm{P}<0.05)$. However, the SAS and SDS scores in group $\mathrm{C}$ after one week of intervention did not differ significantly from those before intervention $(\mathrm{P}>0.05)$. On the whole, the present study demonstrates that psychological counseling online for lung cancer patients during the COVID-19 pandemic can effectively reduce their anxiety and depression scores. In addition, this type of counseling may be an effective psychological counseling method that is worthy of promotion during the pandemic.
\end{abstract}

\section{Introduction}

Coronavirus disease 2019 (COVID-19) is caused by a novel coronavirus named severe acute respiratory syndrome coronavirus 2 (SARS-CoV-2) (1-3). The virus is mainly transmitted via respiratory droplets and human contact of

Correspondence to: Dr Jinyou Li, Department of Thoracic Surgery, Affiliated Hospital of Jiangnan University, 585 North Xingyuan Road, Wuxi, Jiangsu 214000, P.R. China

E-mail:20194032045@stu.suda.edu.cn

Key words: COVID-19, lung cancer, depression, anxiety, online contact with contaminated surfaces $(4,5)$. In general, global population was susceptible to this virus. The symptoms of COVID-19 include cough, fever, diarrhea, shortness of breath and severe pneumonia $(6,7)$. Elderly patients and patients with underlying comorbidities are more prone to the development of severe adverse conditions $(8,9)$. Despite the rapid and large flow of scientific data on possible treatment solutions, none have yet demonstrated unequivocal clinical utility against (COVID-19) (10). Thus, the COVID-19 pandemic has caused a feeling of panic among individuals.

Previous studies have demonstrated that severe infectious diseases can increase anxiety and depression in the general population $(11,12)$. Patients with lung cancer are more likely to develop severe adverse conditions following infection with SARS-CoV-2 due to low immunity and the presence of lung lesions. In addition, lung cancer has the characteristics of a high mortality rate and thus, regular reviewing of the disease and treatment updates are required.

Patients with lung cancer are more likely to suffer from anxiety, depression, and other negative psychological states during the COVID-19 pandemic. In response to this situation, and based on the information provided by Department of Thoracic Surgery, Wuxi Third People's Hospital., the present study was implemented. The present study examined the efficacy of an online psychological counseling method based on WeChat as a communication tool. In addition, the current state of anxiety and depression status of lung cancer patients during the COVID-19 pandemic was assessed, evaluating the effects of online psychological counseling on the reduction of anxiety and depression.

\section{Patients and methods}

Study design and ethical approval. The present study was an experimental intervention study that followed the randomized controlled trial procedure. All participants in the study signed informed consent forms. The Medical Ethics Committee of Wuxi Third People's Hospital (now known as the Affiliated Hospital of Jiangnan University), Wuxi, China approved the study procedures (Approval no. IEC2020020101).

Patients. The subjects included in the present study were patients with pathologically confirmed lung cancer who were required to be followed-up at the Department of Thoracic Surgery, Wuxi Third People's Hospital or hospitalized for 
chemotherapy from February to March, 2020. All the patients involved in the study were not infected with COVID-19.

The inclusion criteria were as follows: i) Patients with lung cancer confirmed by histopathological analysis; ii) patients who needed to be followed-up or inpatients at the Department of Thoracic Surgery, Wuxi Third People's Hospital; iii) patients who had a negative history of viral exposure; and iv) patients who agreed to participate in the study. The exclusion criteria were the following: i) Patients who could not be followed-up (some patients did not wish to be followed-up); ii) patients who had a mental disorder or had recently used psychotropic drugs; and iii) patients who dropped out during the study.

A total of 62 cases were included in the study and they were divided into two groups according to the random number table method. The intervention group (group I) included 30 cases and the control group (group C) included 32 cases. There were no statistically significant differences in age, sex, tumor stage, tumor type and the duration of disease between the two groups (Table I).

Evaluation index. Anxiety was assessed using the Self-Rating Anxiety Scale (SAS) questionnaire and depression was assessed using the Self-Rating Depression Scale (SDS) questionnaire $(13,14)$. The scoring timing was before (following the cooperation of the patient) and one week after the intervention. There were 20 items in the SAS. Each item was divided into four levels according to the feelings of the respondents in the past week and the frequency of symptoms was mainly evaluated. The score of 20 items were added to obtain the total SAS score. The total score was multiplied by 1.25 and the integer part was used to obtain the standard total score (S-SAS). The S-SAS was 50-59 for mild anxiety, 60-69 for moderate anxiety, and $\geq 70$ for severe anxiety. The SDS contains 20 items and its design was based on the diagnostic criteria for depression. Among the 20 items, 10 items are worded positively in relation to symptoms and the other 10 negatively. Each item was divided into four grades. The original total score of SDS (R-SDS) is between 20 and 80 , although the result is usually expressed as an SDS index, which is obtained by expressing the raw score as a 100-point system. A SDS index of 0.5-0.59 indicated mild depression, 0.60-0.69 indicated moderate depression, and an index $\geq 0.07$ indicated severe depression.

Intervention methods. All interventions were completed online through the WeChat platform. The control group only had routine follow-up management, including informing the time of examination or treatment. The intervention group I included not only routine follow-up management, but also psychological counseling. The counseling principles included non-directive intervention, sincerity, empathy, unconditional care and acceptance. Psychological counseling was conducted via face-to-face video, and the counseling time for each patient is $\geq 10 \mathrm{~min}$. The specific methods were as follows: i) Listening carefully during the counseling process. Patients' questions regarding lung cancer or the COVID-19 pandemic were answered patiently. Patient confidence was established based on delivering accurate information to the patients and providing non-directive interventions. ii) Being honest regarding the process of communication, listening carefully to the patient, and communicating with the eyes and expres- sions, and responding to the patient's statements in a timely manner. iii) Through transposition thinking, the aim was to understand the patient's experience due to the extra stress and pressure from both the lung cancer and the pandemic. The aim was to fully understand the patient's anxiety and other negative emotions in order to achieve the purpose of empathy. iv) During the process of communication, in face of the negative emotions of the patients, patients should be treated with compassion, understanding and non-exclusion, so that the patients feel that they are accepted and are not alone.

Statistical analysis. Statistical analysis was performed using SPSS 17.0 statistical software (SPSS, Inc.). The measurement data are expressed as the mean \pm standard deviation (mean $\pm \mathrm{SD}$ ), and the comparison of the mean of the two groups was performed using a Student's t-test. For repeated measures data with interaction effects, mixed-design ANOVA was used. A Chi-squared test was used to compare the ratios and composition ratios. Fisher's exact test was used for cases where the frequency was $<5$. A P-value $<0.05$ was considered to indicate a statistically significant difference.

\section{Results}

The S-SAS in the 62 patients before the intervention was $45.08 \pm 11.05$, including 14 cases with mild anxiety (22.58\%), 7 cases with moderate anxiety (11.29\%) and 1 case with severe anxiety $(1.6 \%)$. The R-SDS was $41.85 \pm 10.20$, including 18 cases with mild depression (29.03\%), 10 cases with moderate depression $(16.13 \%)$ and 4 cases with severe depression $(6.45 \%)$ (Table II). There was no significant difference in the S-SAS and the R-SDS between the two groups before intervention ( $\mathrm{P}>0.05)$ (Table III).

Following one week of intervention, the S-SAS and the R-SDS of the two groups were statistically analyzed. It was found that both the S-SAS and the R-SDS of group I were lower than those of group C $(\mathrm{P}<0.05)$ (Table III).

The S-SAS and the R-SDS of group I after one week of intervention were lower than those before intervention $(\mathrm{P}<0.05)$. The SAS score and SDS score of group $\mathrm{C}$ after one week of intervention did not differ significantly from those before intervention $(\mathrm{P}>0.05)$ (Table III).

\section{Discussion}

At the end of 2019, the COVID-19 pandemic began (15), and this subsequently spread rapidly worldwide. The United States, Italy, Spain, Germany, Iran, France and other countries were severely affected by the pandemic. The World Health Organization declared COVID-19 as a global pandemic on March 11, 2020. COVID-19 and SARS not only have a similar transmission and pathogenic characteristics, but also have similar effects on society and the human body. Previous studies have found that SARS can cause anxiety and depression, as well as other psychological issues in the population $(11,16)$. Similarly, the outbreak of the COVID-19 pandemic has threatened not only human health physically, but also psychologically (17).

At the same time, malignant tumors have become one of the major public health concerns that seriously threaten 
Table I. Demographic characteristics of the study participant and disease-related information.

\begin{tabular}{|c|c|c|c|c|c|c|c|c|c|c|c|}
\hline \multirow[b]{2}{*}{ Group } & \multicolumn{2}{|c|}{ Sex } & \multirow[b]{2}{*}{ Age, years } & \multicolumn{3}{|c|}{ Tumor type } & \multicolumn{4}{|c|}{ Tumor stage } & \multirow[b]{2}{*}{ DOD, months } \\
\hline & M & $\mathrm{F}$ & & LUAD & LUSC & Other & I & II & III & IV & \\
\hline Group I & 9 & 21 & $61.6 \pm 8.16$ & 21 & 8 & 1 & 10 & 13 & 5 & 2 & $5.40 \pm 5.26$ \\
\hline Group C & 11 & 22 & $60.19 \pm 7.10$ & 22 & 9 & 1 & 10 & 16 & 4 & 2 & $4.66 \pm 4.84$ \\
\hline$t / \chi^{2}$ & \multicolumn{2}{|c|}{0.136} & 0.728 & & & & & & & & 0.579 \\
\hline P-value & \multicolumn{2}{|c|}{0.713} & 0.469 & \multicolumn{3}{|c|}{1.00} & \multicolumn{4}{|c|}{0.932} & 0.645 \\
\hline
\end{tabular}

M, male; F, female; LUAD, lung adenocarcinoma; LUSC, lung squamous cell carcinoma; DOD, duration of disease.

Table II. S-SAS and R-SDS scores of all patients prior to intervention, as well as the distribution of various degrees of anxiety or depression in all patients.

Score values and degree of anxiety

Pre-S-SAS $(n=62)$

Pre-R-SDS (n=62)

$\begin{array}{lc}\text { Mean } \pm \text { SD score values } & 45.08 \pm 11.05 \\ \text { Mild } & 14(22.58 \%) \\ \text { Moderate } & 7(11.29 \%) \\ \text { Severe } & 1(1.6 \%) \\ \text { Normal } & 40(64.52 \%) \\ \text { Incidence of anxiety or depression } & 22(35.48 \%)\end{array}$

$41.85 \pm 10.20$

$18(29.03 \%)$

$10(16.13 \%)$

$4(6.45 \%)$

$30(48.39 \%)$

$32(51.61 \%)$

Pre-S-SAS and Pre-R-SDS are the scores prior to intervention. SAS, Self-Rating Anxiety Scale; SDS, Self-Rating Depression Scale.

Table III. Results of statistical analysis of the S-SAS and R-SDS between the two groups before intervention and at one week after intervention.

\begin{tabular}{lcccc}
\hline Scoring system & & & $\begin{array}{c}\text { F value } \\
\text { P-value } \\
\text { (Group I vs. Group C) }\end{array}$ & 0.14 \\
Pre-S-SAS & Group I & Group C & 4.44 & 0.706 \\
7-S-SAS & $45.63 \pm 11.45$ & $44.56 \pm 10.82$ & & 0.034 \\
F value (Pre-S-SAS vs. 7-S-SAS) & $41.00 \pm 8.19$ & $45.84 \pm 9.33$ & 1.667 & 0.03 \\
P-value (Pre-S-SAS vs. 7-S-SAS) & 28.048 & 0.001 & 0.202 & 6.48 \\
Pre-R-SDS & $42.10 \pm 9.54$ & $41.63 \pm 10.92$ & & 0.856 \\
7-R-SDS & $37.27 \pm 6.82$ & $43.06 \pm 10.57$ & & 0.013 \\
F value (Pre-R-SDS vs. 7-R-SDS) & 25.361 & 2.393 & 0.127 & \\
P-value (Pre-R-SDS vs. 7-R-SDS) & 0.001 & & & \\
\hline
\end{tabular}

Pre-S-SAS and Pre-R-SDS are the scores before intervention.7-S-SAS and 7-R-SDS are the score after 7 days of intervention. SAS, Self-Rating Anxiety Scale; SDS, Self-Rating Depression Scale.

human health worldwide. The succumbing of patients due to malignant tumors has become one of the major causes of human mortality $(18,19)$. Some studies have found that patients with malignant tumors are prone to psychological issues, such as anxiety and depression (20-22). Therefore, psychological issues, such as anxiety and depression in patients with malignant tumors are more prominent during the COVID-19 pandemic. Since lung cancer is a malignant tumor associated with the highest morbidity and mortality (23), the analysis of such a patient cohort with regards to the pandemic and adverse psychological effects was considered of interest.

Some studies have found that psychological distress can be reduced by maintaining contact with peers through social networks. Online resources, such as information about mental health education and preventive measure, video-counseling, telemedicine and telepsychiatry services, may prove useful in reducing the psychosocial effects of COVID-19 (24). Some psychologists have exhibited positivity towards the 
use of technologies and web-based interventions to assist patients with mental health issues (25). Some researchers have suggested that online counseling is an effective method with which to provide counseling services during the COVID-19 outbreak (26). Therefore, the aim of the present study was to evaluate the effects of the psychological online counseling of lung cancer patients during the COVID-19 pandemic. The present study evaluated whether online psychological counseling can reduce anxiety and depression in lung cancer patients during the COVID-19 pandemic. Due to the fact that after a period of seven days, a number of patients may not be able to continue to participate in the study, the evaluation time period was set to seven days following the intervention. The SAS questionnaire does not include any questions regarding patients' access to COVID-19-related information, apart from psychological counseling.

According to the present study, during the pandemic period, the incidence of anxiety in lung cancer patients reached $35.48 \%$, while the incidence of depression reached $51.61 \%$ (Table II). This result is much higher than that of $13.1 \%$ during the non-pandemic period (27). This indicates that the psychological issues of anxiety and depression are more common in patients with lung cancer during the COVID-19 pandemic. If mental health issues cannot be promptly addressed and treated, they may aggravate physical diseases and may even lead to vicious events, such as suicide $(28,29)$. Therefore, psychological counseling for lung cancer patients during the COVID-19 pandemic is necessary to improve the quality of life of patients and promote their mental health. The sources of anxiety and depression in lung cancer patients during the pandemic period seem to be the following: i) The characteristics of lung cancer, such as the high mortality, treatment-related side-effects and high treatment costs. ii) Patients with lung cancer are afraid to go visit the hospital, as they are concerned that hospital admittance may increase the risk of COVID-19 infection. Thus, they are afraid to visit the hospital as scheduled for treatment or follow-up schedules. Moreover, they are concerned that the failure of regular treatment or follow-up may aggravate the lung cancer. This ambivalence aggravates the negative emotions. iii) Isolation measures limit the space for lung cancer patients and increase the cost of living. Therefore, prior to conducting psychological counseling with patients through WeChat videos, relevant information is collected and prevention measures for COVID-19 are taken. In this manner, the patients' doubts and concerns can be dealt with more effectively during the psychological counseling process.

In the process of psychological counseling, it was found that patients were plagued by the aforementioned three concerns, such as questions and concerns of whether the lung cancer metastasize will metastasize a few days later; how long they can survive without chemotherapy; whether they will become infected with COVID-19 while visiting the hospital; whether they may not survive if infected with COVID-19, as well as questions of when the pandemic will end. When answering these questions, the principles of non-directive intervention, sincerity, empathy, unconditional care and acceptance are followed. Patients are provided with guidance to correctly understand the above-mentioned issues based on scientific knowledge, such as explaining to patients the principle of the chemotherapy cycle in lung cancer, the five-year survival rate of each stage of lung cancer, current treatment methods for lung cancer, methods for the prevention of COVID-19 infection, and explaining that the majority of patients with COVID-19 experience mild disease. The establishment of a good doctor-patient mutual trust relationship based on the afore-mentioned methods, may help patients to accept medical workers' explanations, and may guide patients to identify false information and avoid panic caused by false information. The aim is to encourage patients to avoid false information that could lead to unnecessary panic, and to build patient confidence and trust through managing their psychological fluctuations and alleviating negative emotions towards lung cancer and COVID-19 diagnosis and treatments.

The present study has some limitations that should be mentioned. Due to the pandemic situation and time constraints, the sample size of the study was small, and the online intervention procedures need to be further improved. The authors aim to improve the intervention process in the future and to expand the sample size in future studies.

In conclusion, in the present study, at seven days after such psychological counseling, it was found that the S-SAS and R-SDS scores of the intervention group were significantly lower than those of the control group, and significantly lower than those before the intervention $(\mathrm{P}<0.05)$. It is thus suggested that psychological counseling for lung cancer patients during the COVID-19 pandemic may effectively reduce patient anxiety and depression scores. This indicates that psychological counseling online for lung cancer patients is a psychological counseling method that is worthy of use during the COVID-19 pandemic.

\section{Acknowledgements}

Not applicable.

\section{Funding}

The present study was supported by the Youth Project of Wuxi Municipal Health and Family Planning Commission (grant no. Q201803) and Youth Project of Jiangnan University Public Health Research Center (grant no. JUPH201826).

\section{Availability of data and materials}

The datasets used and/or analyzed during the current study are available from the corresponding author on reasonable request.

\section{Authors' contributions}

WS contributed to the conception and design of the study and performed the data analyses and wrote the manuscript. JL contributed to the conception and design of the study and assisted in performing the analysis with constructive discussions. Both authors (WS and JL) confirm the authenticity of all the raw data. Both authors have read and approved the final manuscript.

\section{Ethics approval and consent to participate}

All participants in the present study signed informed consent. Medical Ethics Committee of Wuxi Third People's Hospital 
approved the study procedures (Approval no. IEC2020020101). All patients agreed on the use of their medical data and publication. Informed written consent was provided by all patients.

\section{Patient consent for publication}

Not applicable.

\section{Competing interests}

The authors declare that they have no competing interests.

\section{References}

1. Wu Z and McGoogan JM: Characteristics of and important lessons from the coronavirus disease 2019 (COVID-19) outbreak in china: summary of a report of 72,314 cases from the chinese center for disease control and prevention. JAMA 323: 1239-1242, 2020.

2. Parvez MK, Jagirdar RM, Purty RS, Venkata SK, Agrawal V, Kumar J and Tiwari N: COVID-19 pandemic: Understanding the emergence, pathogenesis and containment (Review). World Acad Sci J 2: 18, 2020.

3. Yamani LN and Syahrul F: Public health perspective of the COVID-19 pandemic: Host characteristics and prevention of COVID-19 in the community (Review). World Acad Sci J 2: 21, 2020.

4. Gilbert M, Pullano G, Pinotti F, Valdano E, Poletto C, Boëlle PY, D'Ortenzio E, Yazdanpanah Y, Eholie SP, Altmann M, et al: Preparedness and vulnerability of African countries against importations of COVID-19: A modelling study. Lancet 395: 871-877, 2020.

5. Guo YR, Cao QD, Hong ZS, Tan YY, Chen SD, Jin HJ, Tan KS, Wang DY and Yan Y: The origin, transmission and clinical therapies on coronavirus disease 2019 (COVID-19) outbreak - an update on the status. Mil Med Res 7: 11, 2020.

6. Wang Z, Yang B, Li Q, Wen L and Zhang R: Clinical features of 69 cases with coronavirus disease 2019 in Wuhan, China. Clin Infect Dis 71: 769-777, 2020.

7. Chang D, Lin M, Wei L, Xie L, Zhu G, Dela Cruz CS and Sharma L: Epidemiologic and clinical characteristics of novel coronavirus infections involving 13 patients outside Wuhan, China. JAMA 323: 1092-1093, 2020.

8. Deng Y, Liu W, Liu K, Fang YY, Shang J, Zhou L, Wang K, Leng F, Wei S, Chen L and Liu HG: Clinical characteristics of fatal and recovered cases of coronavirus disease 2019 (COVID-19) in Wuhan, China: a retrospective study. Chin Med J (Engl) 133: 1261-1267, 2020.

9. Wu J, Li W, Shi X, Chen Z, Jiang B, Liu J, Wang D, Liu C, Meng Y, Cui L, et al: Early antiviral treatment contributes to alleviate the severity and improve the prognosis of patients with novel coronavirus disease (COVID-19). J Intern Med 288: $128-138,2020$

10. Nitulescu GM, Paunescu H, Moschos SA, Petrakis D, Nitulescu G, Ion GND, Spandidos DA, Nikolouzakis TK, Drakoulis $\mathrm{N}$ and Tsatsakis A: Comprehensive analysis of drugs to treat SARS-CoV-2 infection: Mechanistic insights into current COVID-19 therapies (Review). Int J Mol Med 46: 467-488, 2020.

11. Wu KK, Chan SK and Ma TM: Posttraumatic stress, anxiety, and depression in survivors of severe acute respiratory syndrome (SARS). J Trauma Stress 18: 39-42, 2005.

12. Liu X, Kakade M, Fuller CJ, Fan B, Fang Y, Kong J, Guan Z and Wu P: Depression after exposure to stressful events: Lessons learned from the severe acute respiratory syndrome epidemic. Compr Psychiatry 53: 15-23, 2012.

13. Samakouri M, Bouhos G, Kadoglou M, Giantzelidou A, Tsolaki K and Livaditis M: Standardization of the Greek version of Zung's Self-rating Anxiety Scale (SAS). Psychiatriki 23: 212-220, 2012 [In Modern Greek (1453-)].
14. Zung WW: A Self-Rating Depression Scale. Arch Gen Psychiatry 12: 63-70, 1965.

15. World Health Organisation (WHO): Country \& Technical Guidance - Coronavirus disease (COVID-19). https://www.who.int/ emergencies/diseases/novel-coronavirus-2019/technical-guidance. Accessed July 17, 2020.

16. Moldofsky $\mathrm{H}$ and Patcai J: Chronic widespread musculoskeletal pain, fatigue, depression and disordered sleep in chronic post-SARS syndrome; a case-controlled study. BMC Neurol 11: 37, 2011.

17. Xiao H, Zhang Y, Kong D, Li S and Yang N: Social Capital and Sleep Quality in Individuals Who Self-Isolated for 14 Days During the Coronavirus Disease 2019 (COVID-19) Outbreak in January 2020 in China. Med Sci Monit 26: e923921, 2020.

18. Arnold M, Rutherford MJ, Bardot A, Ferlay J, Andersson TM, Myklebust TA, Tervonen H, Thursfield V, Ransom D, Shack L, et al: Progress in cancer survival, mortality, and incidence in seven high-income countries 1995-2014 (ICBP SURVMARK-2): A population-based study. Lancet Oncol 20: 1493-1505, 2019.

19. Global Burden of Disease Cancer Collaboration, Fitzmaurice C, Abate D, Abbasi N, Abbastabar H, Abd-Allah F, Abdel-Rahman O, Abdelalim A, Abdoli A, Abdollahpour I, et al: Global, Regional, and National Cancer Incidence, Mortality, Years of Life Lost, Years Lived With Disability, and Disability-Adjusted Life-Years for 29 Cancer Groups, 1990 to 2017: A Systematic Analysis for the Global Burden of Disease Study. JAMA Oncol 5: 1749-1768, 2019.

20. Pitman A, Suleman S, Hyde N and Hodgkiss A: Depression and anxiety in patients with cancer. BMJ 361: k1415, 2018.

21. Niedzwiedz CL, Knifton L, Robb KA, Katikireddi SV and Smith DJ: Depression and anxiety among people living with and beyond cancer: A growing clinical and research priority. BMC Cancer 19: 943, 2019.

22. Mitchell AJ, Chan M, Bhatti H, Halton M, Grassi L, Johansen C and Meader N: Prevalence of depression, anxiety, and adjustment disorder in oncological, haematological, and palliative-care settings: A meta-analysis of 94 interview-based studies. Lancet Oncol 12: 160-174, 2011

23. Raz DJ, Sun V, Kim JY, Williams AC, Koczywas M, Cristea M, Reckamp K, Hayter J, Tiep B and Ferrell B: Long-Term Effect of an Interdisciplinary Supportive Care Intervention for Lung Cancer Survivors After Surgical Procedures. Ann Thorac Surg 101: 495-502, discussion 502-503, 2016.

24. Deolmi M and Pisani F: Psychological and psychiatric impact of COVID-19 pandemic among children and adolescents. Acta Biomed 91: e2020149, 2020.

25. Dores AR, Geraldo A, Carvalho IP and Barbosa F: The use of new digital information and communication technologies in psychological counseling during the COVID-19 pandemic. Int J Environ Res Public Health 17: 7663, 2020.

26. Situmorang DDB: Online/Cyber Counseling Services in the COVID-19 Outbreak: Are They Really New? J Pastoral Care Counsel 74: 166-174, 2020.

27. Walker J, Hansen CH, Martin P, Symeonides S, Ramessur R, Murray G and Sharpe M: Prevalence, associations, and adequacy of treatment of major depression in patients with cancer: A cross-sectional analysis of routinely collected clinical data. Lancet Psychiatry 1: 343-350, 2014.

28. Henson KE, Brock R, Charnock J, Wickramasinghe B, Will O and Pitman A: Risk of suicide after cancer diagnosis in England. JAMA Psychiatry 76: 51-60, 2019.

29. Wang SM, Chang JC, Weng SC, Yeh MK and Lee CS: Risk of suicide within 1 year of cancer diagnosis. Int J Cancer 142: 1986-1993, 2018

This work is licensed under a Creative Commons Attribution-NonCommercial-NoDerivatives 4.0 International (CC BY-NC-ND 4.0) License. 